Supporting Information for:

\title{
Dehydration Pathways of Gypsum and Rehydration Mechanism of Soluble Anhydrite $\gamma-\mathrm{CaSO}_{4}$
}

\author{
Yongbo Tang a , Jianming Gao ${ }^{\mathrm{a}, \mathrm{b}^{*}}$, Chuanbei Liu ${ }^{\mathrm{a}}$, Xuemei Chen ${ }^{\mathrm{a}}$, Yasong Zhao ${ }^{\mathrm{a}}$ \\ ${ }^{\text {a }}$ School of Materials Science and Engineering, Southeast university, Nanjing, 211189, China \\ bJiangSu Key Laboratory of Construction Materials, Nanjing, 211189, China \\ * Corresponding author. Email: jmgao@seu.edu.cn. Tel.: +8613505185020.
}




\section{Matlab programs for plotting Figure 3. (a)}

$[\mathrm{n}, \mathrm{T}]=$ meshgrid $(0: 0.01: 1,273: 0.1: 393)$;

PH2O_dehydration $=\exp \left(\left(1.112^{*} 10^{\wedge}(-5)^{*} T .^{\wedge} 2-1.0^{*} n .^{*}\left(2.35^{\star} 10^{\wedge}(-5)^{*} \mathrm{~T} .{ }^{\wedge} 2-0.1613^{*} \mathrm{~T}+\right.\right.\right.$ $\left.71.2)-0.3069^{*} T+2.439^{*} 10^{\wedge}(-8)^{*} T .^{\wedge} 3+5.466^{*} n .^{\wedge} 2+118.3\right) . /\left(T . *\left(0.008314^{*} n-\right.\right.$ $0.01663))$ );

$\mathrm{PH} 2 \mathrm{O}$-water $=10^{*} 10^{\wedge}-6^{*} \exp \left(6.4127+7.2728^{*} 10^{\wedge}-2^{*}(\mathrm{~T}-273)-2.9858^{*} 10^{\wedge}-4^{*}(\mathrm{~T}-\right.$

273). $\left.{ }^{\wedge} 2+1.0590^{*} 10^{\wedge}-6^{*}(T-273){ }^{\wedge} 3-2.6931^{*} 10^{\wedge}-9^{*}(T-273){ }^{\wedge} 4+3.3553^{*} 10^{\wedge}-12^{*}(T-273) . \wedge 5\right)$;

Patm $=0 * n+1$

mesh(n, T,Patm,'FaceColor','r','EdgeColor','none');

alpha(0.7)

hold on

surf(n,T,PH2O_water,'FaceColor','b','EdgeColor','none');

alpha(0.5)

surf(n,T,PH2O_dehydration,'FaceColor','g','EdgeColor','none');

tol= $\operatorname{diff}(z \lim )^{*} 0.001$;

$\mathrm{r} 0=(\operatorname{abs}($ Patm-PH2O_dehydration $)<=$ tol $) \& n<=0.61 ;$

$\mathrm{zz}=\left(\right.$ Patm $+\mathrm{PH} 2 \mathrm{O} \_$dehydration $) / 2$;

plot3(n(r0),T(r0),zz(r0),'-y','linewidth',1)

tol $=\operatorname{diff}(\mathrm{zlim})^{*} 0.0001$

$\mathrm{r} 0=\left(\operatorname{abs}\left(\mathrm{PH} 2 \mathrm{O} \_\right.\right.$dehydration- $\mathrm{PH} 2 \mathrm{O} \_$water $)<=$tol $) \& \mathrm{n}>=0.61$; 
$\mathrm{zz}=\left(\mathrm{PH} 2 \mathrm{O} \_\right.$water+PH2O_dehydration $) / 2$;

plot3(n(r0),T(r0),zz(r0),'-w','linewidth',1)

text(0.3,240,0,'n/mol','rotation', 19);

text(-0.2,330,0,'T/K','rotation',-33);

text(0.1,420,2,'P/atm','rotation', 90$) ; \square \square$

Where PH2O_dehydration is the equilibrium water vapor pressure in $\mathrm{CaSO}_{4} \cdot 2 \mathrm{H}_{2} \mathrm{O}$ decomposing into $\mathrm{CaSO}_{4} \cdot \mathrm{nH}_{2} \mathrm{O}$; $\mathrm{PH} 2 \mathrm{O}$ _water represents the saturated vapor pressure; $\mathrm{n}$ denotes the value of combined water content of $\mathrm{CaSO}_{4} \cdot \mathrm{nH}_{2} \mathrm{O}$.

2. The lowest energy conformation of $\mathrm{CaSO}_{4} \bullet 0.67 \mathrm{H}_{2} \mathrm{O}$ phase

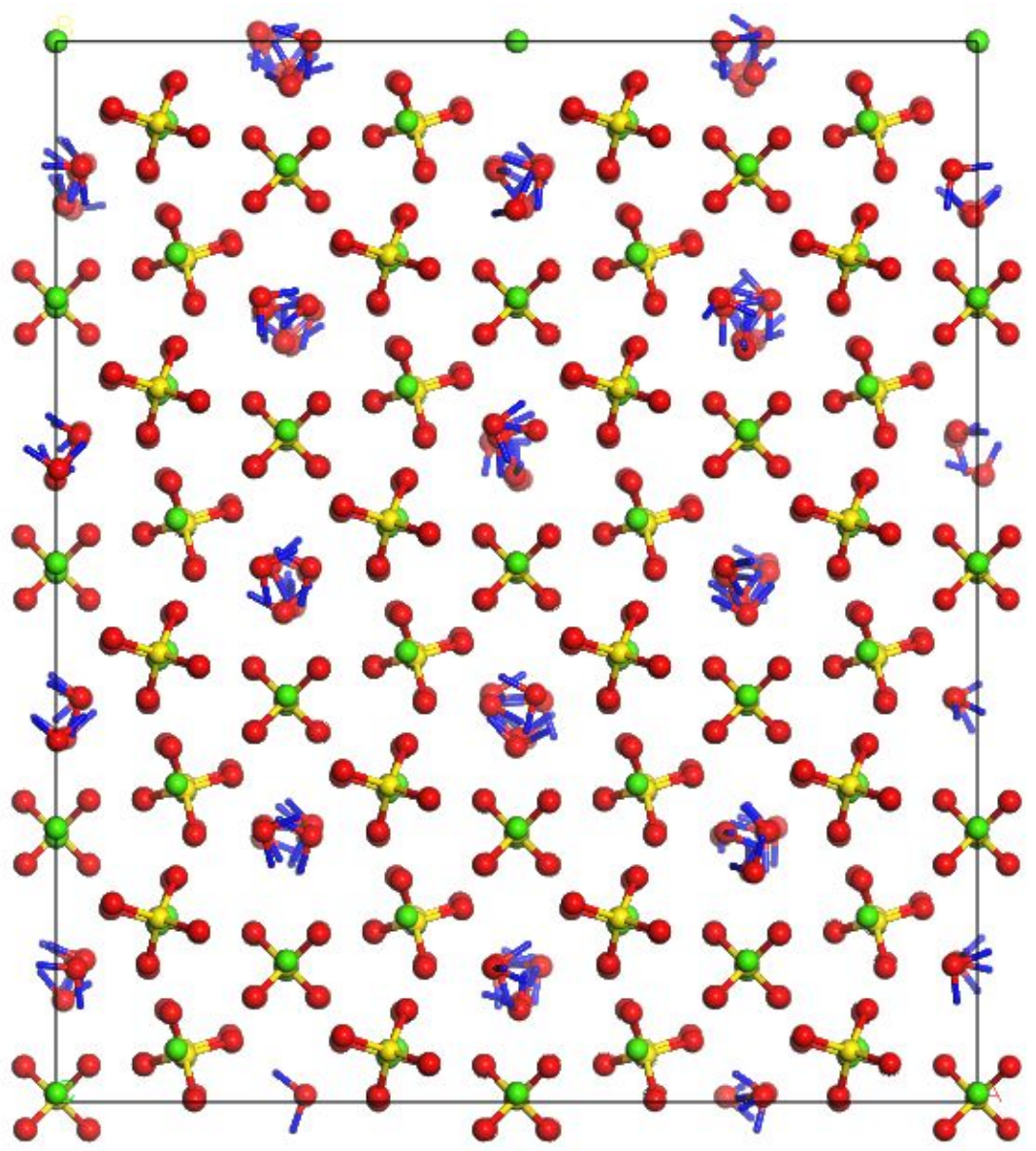


Figure S1. The lowest energy conformation of $\mathrm{CaSO}_{4} \cdot 0.67 \mathrm{H}_{2} \mathrm{O}$ phase viewed along [001] according to structural model determined by Monte Carlo simulation.

Monte Carlo simulation results indicate that the value of the combined water number can range from 0 to 0.67 with the increase in water vapor partial pressure. There are four water molecules were distributed among one channel in the unit cell, the occupancy of the four sites per channel in the unit cell corresponds to a stoichiometry $\mathrm{CaSO}_{4} \cdot 0.67 \mathrm{H}_{2} \mathrm{O}$ phase. 